\title{
W Physics at LEP
}

\section{Monica Pepe Altarelli}

INFN - Laboratori Nazionali di Frascati and CERN, EP Division

E-mail: Minica.Pepe.AItarelli@iCERN.CH'

ABSTRACT: A summary of the W-boson properties measured by the four LEP collaborations is presented here. These properties are updated to take into account the most recent results presented at the ICHEP98 Conference.

\section{WW cross sections and W branch- ing fractions}

After the period of running at the $\mathrm{Z}$, the centreof-mass energy of LEP has been progressively increased from $161 \mathrm{GeV}$, i.e., just above the $\mathrm{W}$ pair production threshold to the current centreof-mass energy of $189 \mathrm{GeV}$. Each LEP experiment has collected a luminosity of approximately $10 \mathrm{pb}^{-1}$ at $\sqrt{s}=161 \mathrm{GeV}, 10 \mathrm{pb}^{-1}$ at $\sqrt{s}=$ $172 \mathrm{GeV}$ and $55 \mathrm{pb}^{-1}$ at $\sqrt{s}=183 \mathrm{GeV}$. The current run at $\sqrt{s}=189 \mathrm{GeV}$ is expected to yield a luminosity of $\sim 150 \mathrm{pb}^{-1}$; preliminary results on the $\mathrm{W}^{+} \mathrm{W}^{-}$cross section at $\sqrt{s}=189 \mathrm{GeV}$ based on the data analysed for ICHEP98 (i.e., about $36 \mathrm{pb}^{-1}$ per experiment) are also reported.

To lowest order, three Feynman diagrams contribute to W pair production at LEP II, the $s$ channel $\mathrm{Z}$ and $\gamma$ exchange and the $t$-channel $\nu_{e}$ exchange (the so-called CC03 diagrams displayed in Figure $\underline{1}_{1}^{\overline{1}}$ for the $\mu \nu_{\mu} \mathrm{u} \overline{\mathrm{d}}$ final state). The $s$ -
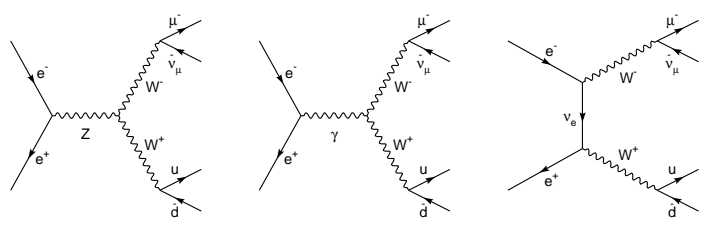

Figure 1: Signal diagrams for $\mathrm{e}^{+} \mathrm{e}^{-} \rightarrow \mu \nu_{\mu} \mathrm{u} \overline{\mathrm{d}}$.

channel diagrams arise as a consequence of the trilinear gauge-boson vertices $\gamma \mathrm{WW}$ and ZWW. The process which is experimentally relevant is $\mathrm{e}^{+} \mathrm{e}^{-} \rightarrow \mathrm{W}^{+} \mathrm{W}^{-} \rightarrow \mathrm{f}_{1} \overline{\mathrm{f}}_{2} \mathrm{f}_{3} \overline{\mathrm{f}}_{4}$. Many more dia- grams contribute to four-fermion production. Those with the same final states as for $\mathrm{W}^{+} \mathrm{W}^{-}$ production interfere with the signal processes. Therefore, to obtain the $\mathrm{W}^{+} \mathrm{W}^{-}$cross sections corresponding to the three CC03 diagrams, the measurements have to be corrected for four-fermion effects.

Table 1 gives the values of the cross sections for the four experiments combined at the differ-

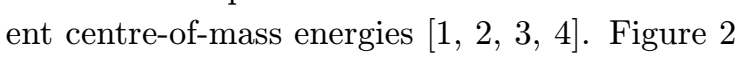
shows the measured cross sections as a function of centre-of-mass energy together with the Standard Model (SM) prediction (full line). Also shown are the predictions obtained assuming the existence of the $t$-channel $\nu_{e}$-exchange diagram only (dotted line) or if the ZWW vertex did not exist (dashed line). While contributions from the individual Feynman graphs grow with energy, an energy behaviour in agreement with data is only obtained when the full amplitude is considered, due to cancellations which can be traced to the gauge theory relations between fermion-

Table 1: $\mathrm{W}^{+} \mathrm{W}^{-}$cross sections for the four $L E P$ experiments combined for the various centre-of-mass energies. The results relative to the data taken at $\sqrt{s}=183 \mathrm{GeV}$ and $189 \mathrm{GeV}$ are still preliminary.

\begin{tabular}{|c|c|}
\hline$\sqrt{s}(\mathrm{GeV})$ & $\sigma_{\mathrm{W}^{+} \mathrm{W}^{-}}(\mathrm{pb})$ \\
\hline \hline 161 & $3.69 \pm 0.45$ \\
172 & $12.05 \pm 0.73$ \\
183 & $15.86 \pm 0.40$ \\
189 & $15.24 \pm 0.57$ \\
\hline
\end{tabular}




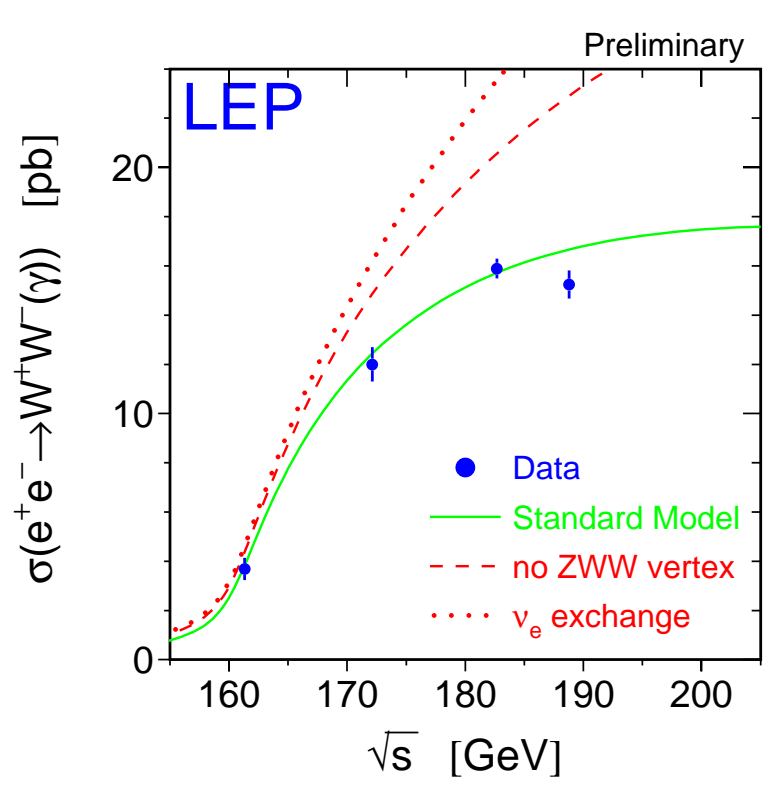

Figure 2: $W$-pair cross section as a function of $\sqrt{s}$. The data points are the combined LEP cross sections. The curves show the Standard Model prediction (full line), the calculated cross section if no $Z W W$ vertex existed (dashed line) and if both $Z W W$ and $\gamma W W$ vertices did not exist (dotted line).

gauge boson vertices and triple gauge couplings.

In Table $\overline{2}$, , the $\mathrm{W}$ decay branching ratios are reported with and without assuming lepton uni-

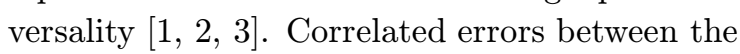
various channels are taken into account in the measurements; in particular the branching ratio for the channel $\mathrm{W} \rightarrow \tau \nu_{\tau}$ has a correlation of $25 \%$ with the other two leptonic channels.

The $\mathrm{W}$ hadronic branching ratio $\mathrm{B}_{\mathrm{h}}$ can be related to the six elements of the CKM matrix $\left(\mathrm{V}_{\mathrm{CKM}}\right)$ not involving the top quark via the formula

$$
\frac{B_{h}}{1-B_{h}}=\sum_{i=u, c}\left|V_{i, j=d, s, b}\right|^{2}\left(1+\frac{\alpha_{s}}{\pi}\right) .
$$

Since $\left|\mathrm{V}_{\mathrm{cs}}\right|$ is rather poorly measured $\left(\left|\mathrm{V}_{\mathrm{cs}}\right|=\right.$ $1.04 \pm 0.16$ from data on branching ratios for $D_{e 3}$ and D lifetimes (15inj) it can be determined from the above expression by taking for the other CKM matrices the current world averages
Table 2: Summary of $W$ branching fractions from $\mathrm{W}^{+} \mathrm{W}^{-}$cross section measurements up to $183 \mathrm{GeV}$

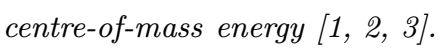

\begin{tabular}{|c|c|c|c|c|}
\hline Exp. & $\begin{array}{c}\mathrm{W} \rightarrow \mathrm{e} \nu \\
(\%)\end{array}$ & $\begin{array}{c}\mathrm{W} \rightarrow \mu \nu \\
(\%)\end{array}$ & $\begin{array}{c}\mathrm{W} \rightarrow \tau \nu \\
(\%)\end{array}$ & $\begin{array}{c}W \rightarrow \text { hadrons } \\
(\%)\end{array}$ \\
\hline $\bar{A}$ & $11.2 \pm 0.8 \pm 0.3$ & $9.9 \pm 0.8 \pm 0.2$ & $9.7 \pm 1.0 \pm 0.3$ & $69.0 \pm 1.2 \pm 0.6$ \\
\hline D & $9.9 \pm 1.1 \pm 0.5$ & $11.4 \pm 1.1 \pm 0.5$ & $11.2 \pm 1.7 \pm 0.7$ & $67.5 \pm 1.5 \pm 0.9$ \\
\hline $\mathrm{L}$ & $10.5 \pm 0.9 \pm 0.2$ & $10.2 \pm 0.9 \pm 0.2$ & $9.0 \pm 1.2 \pm 0.3$ & $70.1 \pm 1.3 \pm 0.4$ \\
\hline 0 & $11.7 \pm 0.9 \pm 0.3$ & $10.1 \pm 0.8 \pm 0.3$ & $10.3 \pm 1.0 \pm 0.3$ & $67.9 \pm 1.2 \pm 0.6$ \\
\hline LEP & $10.92 \pm 0.49$ & $10.29 \pm 0.47$ & $9.95 \pm 0.60$ & $68.79 \pm 0.77$ \\
\hline $\begin{array}{l}\mathrm{LEP} \\
\mathrm{W} \rightarrow \ell \nu\end{array}$ & & $10.40 \pm 0.26$ & & \\
\hline SM & & 10.8 & & 67.5 \\
\hline
\end{tabular}

$\alpha_{s}=0.118 \pm 0.03$ [isin and not assuming unitarity of $\mathrm{V}_{\mathrm{CKM}}$. The result is [1]1,

$$
\left|\mathrm{V}_{\text {cs }}\right|=1.04 \pm 0.04 \text {. }
$$

The error on this result is dominated by the statistical error on the $\mathrm{W}$ branching fractions. The element $\left|V_{c s}\right|$ can also be determined by direct flavour tagging, based, for example, on a lifetime or $\mathrm{D}^{*}$ tag. The result is however less precise in

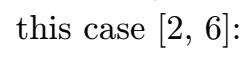

$$
\left|\mathrm{V}_{\mathrm{cs}}\right|=0.99 \pm 0.11
$$

\section{Triple gauge couplings}

The most general Lorentz invariant Lagrangian describing the triple gauge boson interactions has fourteen terms which reduce to five assuming electromagnetic gauge invariance as well as $\mathrm{P}$ and $\mathrm{C}$ conservation. Since we are interested in possible deviations from the SM, the anomalous couplings $\Delta g_{1}{ }^{Z}, \Delta k_{Z}, \Delta k_{\gamma}, \lambda_{Z}$ and $\lambda_{\gamma}$, which are all zero in the SM at tree level, are chosen as free parameters. The Triple Gauge boson Couplings ( TGCs) contribute via loops to observables which are precisely measured at LEP I. It is therefore convenient to choose combinations of couplings not tightly constrained by existing LEP I data. This leads to the choice at LEP II

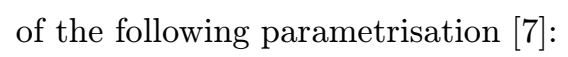

$$
\begin{aligned}
\alpha_{\mathrm{W} \phi} & =\Delta g_{1}{ }^{Z} \cos ^{2} \theta_{\mathrm{W}} \\
\alpha_{\mathrm{W}} & =\lambda_{\gamma} \\
\alpha_{\mathrm{B} \phi} & =\Delta \kappa_{\gamma}-\Delta g_{1}{ }^{Z} \cos ^{2} \theta_{\mathrm{W}}
\end{aligned}
$$


with the constraints

$\lambda_{\mathrm{Z}}=\lambda_{\gamma}$ and $\Delta \kappa_{\mathrm{Z}}=-\Delta \kappa_{\gamma} \tan ^{2} \theta_{\mathrm{W}}+\Delta \mathrm{g}_{1} \mathrm{Z}$

if $\mathrm{SU}(2) \otimes \mathrm{SU}(1)$ gauge invariance is also required. The $\alpha$-parameters are all zero in the SM at tree level.

Anomalous couplings increase the $\mathrm{W}^{+} \mathrm{W}^{-}$ cross section and change the angular distribution of the produced $\mathrm{W}$ bosons and of their decay products. The strongest information is provided by the semileptonic $\mathrm{q} \overline{\mathrm{q}} \ell \nu$ decay for which the charge assignment is unambiguous. On the contrary, in the case of the hadronic channel, without quark charge or flavour tagging, the fermion and anti-fermion cannot be distinguished. The differential cross sections in the production and decay angles are written in terms of the $\mathrm{W}$ helicity amplitudes, which are, in turn, well defined functions of the TGCs.

Additional sensitivity to the $\mathrm{WW} \gamma$ couplings is provided by single photon and especially single $\mathrm{W}$ events. The dominant diagrams for single $\mathrm{W}$ production in the $\mathrm{e} \nu \mu \nu$ final state are shown in Figure $\overline{\beta_{i}}$, the first diagram being the one providing sensitivity to the $\mathrm{WW} \gamma$ vertex. Since, typi-

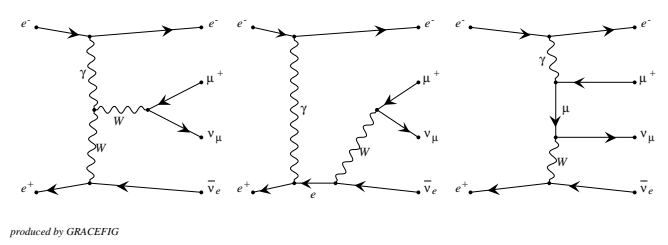

Figure 3: Dominant diagrams for the $\mathrm{e}^{+} \mathrm{e}^{-} \rightarrow$ (e) $\nu \mu \nu$ final state.

cally, the electron goes down the beam pipe, the signatures are a single energetic lepton, or two acoplanar jets and large missing energy. Figure shows the gain in sensitivity on $\Delta \kappa_{\gamma}$ (and therefore on $\alpha_{\mathrm{B} \phi}$ ) obtained when the "standard" WW analysis is combined with the single W analysis [i,

The results of fits to $\alpha_{\mathrm{W} \phi}, \alpha_{\mathrm{W}}$ and $\alpha_{\mathrm{B} \phi}$ are shown in Figure $\overline{5}$, , assuming in each case that the other two anomalous couplings are zero.

The combination is performed by adding the loglikelihood curves supplied by the LEP and D0 [9.9] experiments. The one standard deviation and
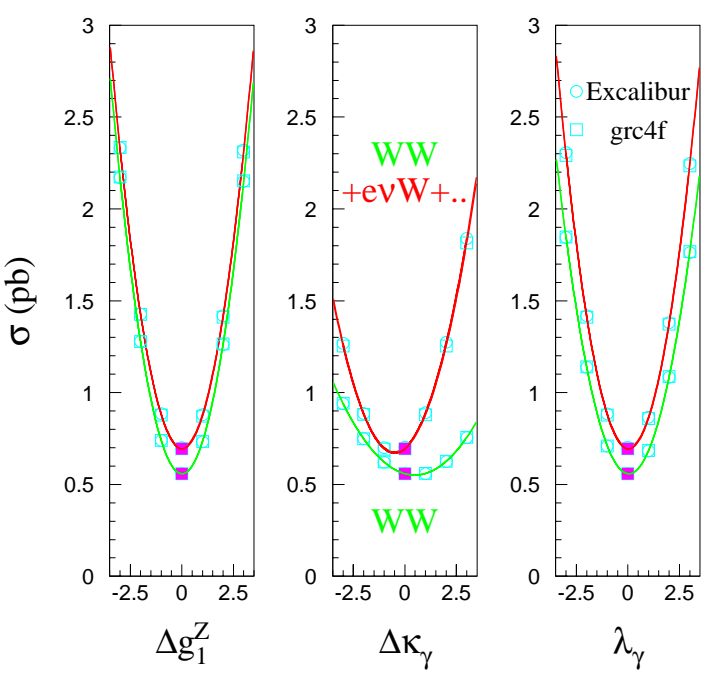

Figure 4: Sensitivity of the cross sections to $\Delta g_{1}{ }^{Z}$, $\Delta \kappa_{\gamma}$ and $\lambda_{\gamma}$ with and without inclusion of the single $W$ processes.

95\% confidence limits are taken as the parameter values where $-\Delta \log \mathrm{L}=0.5$ and 1.92 , respectively. No discrepancy with the SM is observed, however the accuracy on the determination of the $\alpha$ parameter is rather poor (in the SM, from radiative corrections, they are expected to be of order $\left.10^{-2}, 10^{-3}\right)$.

\section{Measurement of the $\mathrm{W}$ mass}

The data collected at centre-of-mass energy of $161 \mathrm{GeV}$, i.e., just above the $\mathrm{W}$ pair production threshold, were used to obtain $M_{\mathrm{W}}$ by comparing the measured cross section with a theoretical calculation which has $M_{\mathrm{W}}$ as a free parameter. In fact, for $\sqrt{s} \simeq 2 M_{\mathrm{W}}$, the value of the cross section is very sensitive to the $\mathrm{W}$ mass and the precise value of $161 \mathrm{GeV}$ was chosen as the best compromise between sensitivity to $M_{\mathrm{W}}$ and statistics. Despite the fact that one relies on a theoretical calculation based on the SM to derive $M_{\mathrm{W}}$, the model dependence is small since at threshold the cross section is dominated by the well established $\nu_{\mathrm{e}} t$-channel exchange diagram. From the LEP combined cross section at $161 \mathrm{GeV}$ $\sigma_{\mathrm{WW}}=3.69 \pm 0.45 \mathrm{pb}$, the following result for 


\section{LEP+DO COMBINATION}
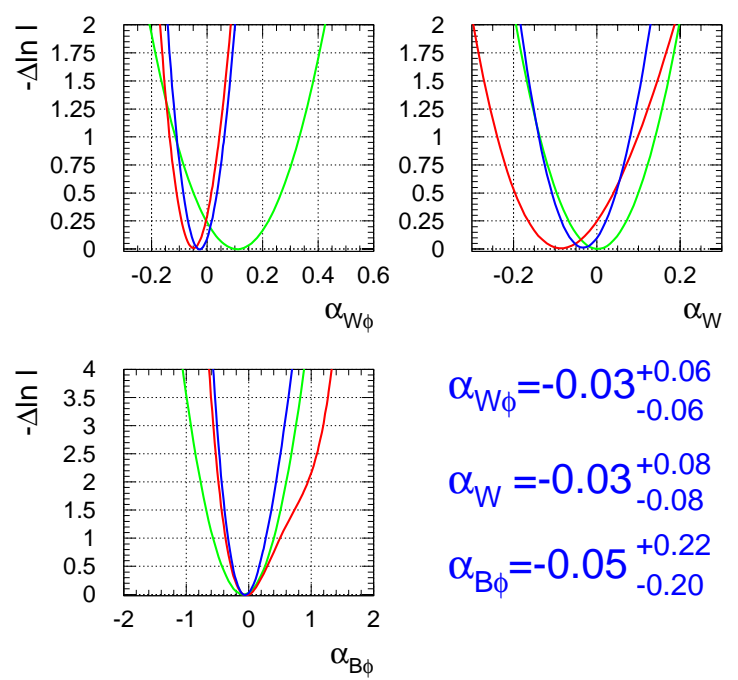

$\alpha_{W_{\phi}}=-0.03_{-0.06}^{+0.06}$

$\alpha_{W}=-0.03_{-0.08}^{+0.08}$

$\alpha_{\mathrm{B} \phi}=-0.05_{-0.20}^{+0.22}$

Figure 5: Results for the three $\alpha$ couplings combining LEP (dark grey line), DO (light grey line) and $L E P+D O$ (black line).

$M_{\mathrm{W}}$ is obtained:

$$
M_{\mathrm{W}}=80.40 \pm 0.22 \mathrm{GeV} .
$$

At higher energies $M_{\mathrm{W}}$ is obtained by direct reconstruction of the jet-jet invariant masses from the channels:

$$
\begin{aligned}
& \mathrm{W}^{+} \mathrm{W}^{-} \rightarrow \mathrm{q} \overline{\mathrm{q}} \mathrm{q} \overline{\mathrm{q}} \quad(\sim 45 \% \text { of cases }) \\
& \mathrm{W}^{+} \mathrm{W}^{-} \rightarrow \mathrm{q} \overline{\mathrm{q}} \ell \nu \quad(\sim 44 \% \text { of cases }) .
\end{aligned}
$$

These topologies are selected with high efficiency and low background. Efficiency and purity for the fully hadronic events are approximately $85 \%$. The semileptonic events are selected with typically $85 \%$ ( $60 \%$ ) efficiency and 95\% ( $85 \%$ ) purity for e and $\mu(\tau)$. The purely leptonic channel has not been used so far due to the lack of sufficient constraints on the kinematics of the event, as it contains at least two neutrinos.

A kinematic fit requiring energy and momentum conservation is used to improve the invariant mass resolution. An additional constraint based on the equality of the two $\mathrm{W}$ masses in an event is also frequently used. In the four-jet case, three kinematic fits are performed for the three possible jet-jet pairings and the resulting fit probabil-
Table 3: Summary of $W$ mass measurements by direct reconstruction (i.e. using data at $\sqrt{s}=172$ and $183 \mathrm{GeV}$ ) for the four LEP experiments in the vari-

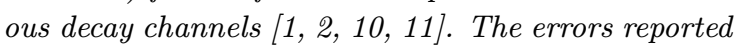
include both statistical and systematic uncertainties.

\begin{tabular}{|l|c|c|c|}
\hline Exp. & $\begin{array}{c}\text { Semileptonic } \\
M_{\mathrm{W}}(\mathrm{GeV})\end{array}$ & $\begin{array}{c}\text { Hadronic } \\
M_{\mathrm{W}}(\mathrm{GeV})\end{array}$ & $\begin{array}{c}\text { Combined } \\
M_{\mathrm{W}}(\mathrm{GeV})\end{array}$ \\
\hline ALEPH & $80.34 \pm 0.18$ & $80.53 \pm 0.18$ & $80.44 \pm 0.13$ \\
DELPHI & $80.50 \pm 0.24$ & $80.01 \pm 0.22$ & $80.24 \pm 0.17$ \\
L3 & $80.09 \pm 0.24$ & $80.59 \pm 0.23$ & $80.40 \pm 0.18$ \\
OPAL & $80.29 \pm 0.19$ & $80.40 \pm 0.24$ & $80.34 \pm 0.15$ \\
\hline Combined & $80.31 \pm 0.11$ & $80.39 \pm 0.14$ & $80.36 \pm 0.09$ \\
\hline
\end{tabular}

ities are used to discard, in general, at least one of the three combinations.

The invariant mass distribution has a BreitWigner shape which is distorted by several effects such as initial state radiation, detector resolution, misassignement of particles between the two W bosons, background, analysis biases, etc, which can only be evaluated by extensive Monte Carlo simulations. What is generally done to extract $M_{\mathrm{W}}$ is to compare the measured invariant mass spectra with the corresponding distributions from simulated experiments based on different input $\mathrm{W}$ masses. To avoid generating large Monte Carlo samples at many different masses, starting from a few reference input $\mathrm{W}$ masses, the mass spectra corresponding to other choices of $M_{\mathrm{W}}$ are obtained by reweighting each event in the reference Monte Carlo by the ratio of cross sections calculated with the new and the reference $\mathrm{W}$ mass.

Table $\overline{3}-1$ shows a summary of the $\mathrm{W}$ mass measurement by direct reconstruction (at 172 and $183 \mathrm{GeV}$ ) for the four LEP experiments in the various decay modes. The combined four-experiment

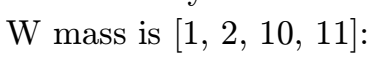

$$
\begin{aligned}
M_{\mathrm{W}}^{\text {lept }}(172-183) & =80.31 \pm 0.10_{\text {stat }} \pm 0.03_{\text {syst }} \pm 0.025_{\mathrm{LEP}} \\
M_{\mathrm{W}}^{\text {had }}(172-183) & =80.39 \pm 0.093_{\text {stat }} \pm 0.05_{\text {syst }} \pm 0.09_{\mathrm{FSI}} \pm 0.025_{\mathrm{LEP}} \\
M_{\mathrm{W}}(172-183) & =80.36 \pm 0.08 \pm 0.05_{\mathrm{FSI}} \pm 0.025_{\mathrm{LEP}} .
\end{aligned}
$$

The semileptonic and hadronic channels have comparable branching ratios and selection efficiencies and give comparable mass resolution. However, the hadronic mode has an additional systematic error of $90 \mathrm{MeV}$ associated to final state interaction effects (FSI) which represents the largest source of systematic uncertainty, as shown in Ta- 
Table 4: "Typical" systematic uncertainties for the semileptonic and hadronic channels. The upper (lower) part of the Table gives those errors which are not correlated (correlated) between experiments.

\begin{tabular}{|l|c|c|}
\hline $\begin{array}{l}\text { Systematic } \\
\text { source }\end{array}$ & $\begin{array}{c}\text { Semileptonic } \\
\delta M_{\mathrm{W}}(\mathrm{MeV})\end{array}$ \\
\hline Detector calibration & 40 & 30 \\
QCD background & & 10 \\
MC statistics & 10 & 10 \\
\hline Hadronisation & 25 & 30 \\
ISR & 15 & 15 \\
Beam energy & 25 & 25 \\
Final State Interactions & - & 90 \\
\hline TOTAL & $\sim 60$ & 110 \\
\hline
\end{tabular}

ble $\overline{4}$. Final state interactions may arise since the separation of the $\mathrm{W}$ decay vertices at LEP II is $\sim 0.1 \mathrm{fm}$, a distance small with respect to the typical hadronisation scale of $\sim 1 \mathrm{fm}$ (or, in other words, $\left.\Gamma_{\mathrm{W}} \sim 10 \Lambda_{\mathrm{QCD}}\right)$. As a result, interconnection phenomena may obscure the separate identities of the two $\mathrm{W}$ bosons distorting the mass determination in the hadronic channel. These interconnection effects can be associated to colour fields stretched between quark lines from different W bosons ("Colour Reconnection") or to interference between identical bosons close in phase space, but produced by different W decays ("BoseEinstein correlations").

The best test of colour reconnection is realized by comparing mean values of charged particle multiplicity and event-shape distributions in the fully hadronic and semileptonic modes since many systematic effects cancel in the difference. The distributions for the $q \bar{q} q \bar{q}$ mode should be equal to twice the $\mathrm{q} \overline{\mathrm{q}} \ell \nu$ mode after removing the final state lepton or its decay products. The following result on the average charged multiplicity difference between $q \bar{q} q \bar{q}$ and $q \bar{q} \ell \nu$ is obtained by

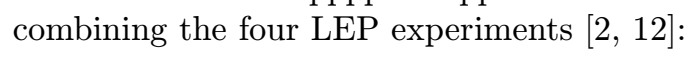

$$
\Delta n_{\mathrm{ch}} \equiv\left\langle n_{\mathrm{ch}}{ }^{\mathrm{q} \overline{\mathrm{q}} \overline{\mathrm{q}}}\right\rangle-2\left\langle n_{\mathrm{ch}}{ }^{\mathrm{q} \overline{\mathrm{q}} \ell \nu}\right\rangle=0.20 \pm 0.50 \text {. }
$$

At the current level of statistical precision, no evidence for colour reconnection effect is found in the observables studied. Most models, such

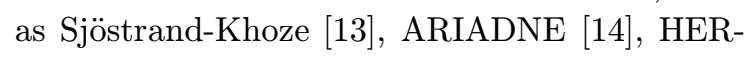
WIG [15], are consistent with the data and predict shifts for $M_{\mathrm{W}}$ smaller than $\sim 50 \mathrm{MeV}$. The Ellis-Geiger model [1] $\left.{ }^{1}\right]$ has not been used to es- timate the systematic error since, in its current implementation, does not reproduce a variety of the measured event shapes.

The simplest method to analyse Bose-Einstein correlations is to measure the ratio of like-sign to unlike-sign pion pairs as a function of $Q^{2}=$ $\left(p_{1}-p_{2}\right)^{2}$ where $p_{1}$ and $p_{2}$ are the particle fourmomenta. Contribution of pion pairs originating from the same W are subtracted statistically, using the distribution obtained from semileptonic events. Bose-Einstein correlations are observed in hadronic and semileptonic $\mathrm{W}$ decays. However, at the present level of statistics, there is no experimental evidence for Bose-Einstein correlations for pairs originating from different $\mathrm{W}$

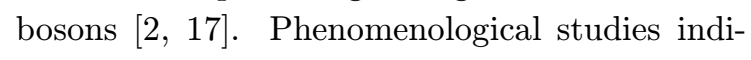
cate that this effect could introduce a shift to $M_{\mathrm{W}}$ smaller than $\sim 50 \mathrm{MeV}$. On the basis of the present experimental and phenomenological results, the error of $90 \mathrm{MeV}$ which is currently assigned as common systematic uncertainty between the LEP experiments due to final state interactions (Bose-Einstein and Colour Reconnection effects) is probably a rather conservative estimate.

Since the systematic uncertainties of the direct reconstruction technique and threshold method are largely independent, the two measurements can be combined, yielding the following result:

$$
M_{\mathrm{W}}=80.37 \pm 0.09 \mathrm{GeV}
$$

Figure '6י-' shows a summary of direct determinations of $M_{\mathrm{W}}$ from LEP and the TEVATRON, as well as the indirect estimates from $\nu \mathrm{N}$ scattering and from radiative corrections using all elec-

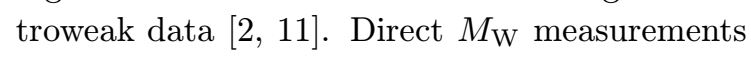
provide a precision which is approaching the one obtained by radiative corrections, allowing a further important test of the SM. The goal of measuring by the end of the LEP II programme in the year 2000 the $\mathrm{W}$ mass with a precision of $\sim 30$ $40 \mathrm{MeV}$ seems to be in reach. Since $M_{\mathrm{W}}$ is an observable which is sensitive to $M_{\mathrm{H}}$, this measurement will allow to put additional constraints on the Higgs mass. The real break-through would be of course the discovery of the Higgs in the $\sim 10 \mathrm{GeV}$ mass window which is still accessible to LEP II. 


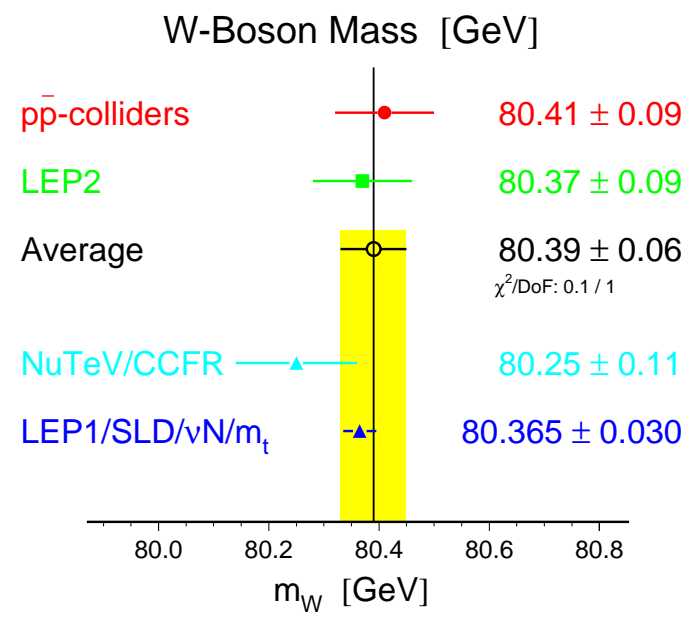

Figure 6: Summary of $M_{\mathrm{W}}$ measurements from $L E P$, the TEVATRON experiments and $\nu N$ scattering as well as the indirect determination derived from all other electroweak data.

\section{References}

[1] LEPEWWG/MW/98-02, LEP WW cross section and $\mathrm{W}$ mass for '98 Summer conferences, The LEP Electroweak W Mass Working Group, available at the following WWW address: http://www.cern.ch/LEPEWWG/wmass/ , and references herein.

[2] D.Karlen, Experimental Status of the Standard Model, Plenary talk presented at ICHEP98.

[3] L.Malgeri, WW Cross Section and Branching Fraction Measurements at LEP, Talk presented at ICHEP98.

[4] The LEP Collaborations ALEPH, DELPHI, L3, OPAL and the LEP Electroweak Working Group and the SLD Heavy Flavour Group, A combination of Preliminary LEP Electroweak Measurements and Constraints on the Standard Model, CERN-PPE/97-154.

[5] R.M.Barnett et al, Review of particle properties, Phys. Rev. D54 (1996) 1.

[6] V.Obraztsov, Measurements of $\left|\mathrm{V}_{\mathrm{cs}}\right|$ in $\mathrm{W}$ decays at LEP II, Talk presented at ICHEP98.

[7] Physics at LEP2, Edited by G.Altarelli, T.Sjöstrand and F.Zwirner, Report on the LEP2 workshop 1995, CERN 96-01 (1996) Vol 1, p.525.

[8] R.Tanaka, Single W Production at LEP2, Talk presented at ICHEP98.
[9] LEPEWWG/TGC/98-01, A Combination of Preliminary Measurements of Triple Gauge Boson Coupling Parameters Measured by the LEP and D0 Experiments, TGC Combination Group, Prepared from Contributions of the LEP and D0 experiments to the 1998 winter conferences. Available at: http://www.cern.ch/LEPEWWG/tgc/ .

[10] H.Przysiezniak, $M_{\mathrm{W}}$ by direct reconstruction at LEP in the semileptonic channel, Talk presented at ICHEP98.

[11] M.Thomson, $M_{\mathrm{W}}$ by direct reconstruction at LEP in the hadronic channel, Talk presented at ICHEP98.

[12] N.K.Watson, Hadronic Decay Properties, Talk presented at ICHEP98.

[13] T.Sjöstrand and V.A.Khoze, Z. Phys. C62 (1994) 281;

T.Sjöstrand and V.A.Khoze, CERN-TH/98-74.

[14] L.Lönnblad, Z. Phys. C70 (1996) 107.

[15] G.Marchesini et al, Comput. Phys. Commun 67 (1992) 465.

[16] J.Ellis and K.Geiger, Phys. Lett. B404 (1997) 230.

[17] R.Moller, Bose-Einstein Correlations in WW events, Talk presented at ICHEP98. 